\title{
Water quality analysis using an inexpensive device and a mobile phone
}

\author{
Timo Toivanen ${ }^{1 *}$, Sampsa Koponen², Ville Kotovirta ${ }^{1}$, Matthieu Molinier ${ }^{1}$ and Peng Chengyuan ${ }^{1}$
}

\begin{abstract}
Background: Water transparency is one indicator of water quality. High water transparency is an indication of clean water. A common method for measuring water transparency is Secchi depth. In this paper, we present an approach to water quality (Secchi depth and turbidity) monitoring using mobile phones and a small device designed for water quality measurements.

Results: The water quality parameters were analysed automatically from the images taken using mobile phone cameras. During the summer of 2012, we conducted a field trial in which 100 test users gathered 1,146 observations using the system. The results of the automatic Secchi3000 depth analysis were compared against reference measurements, and they indicate that our approach can be used for quantitative water quality measurements.
\end{abstract}

Conclusions: Results show that overall the system performs well. Both Secchi depth and turbidity are estimated with excellent or good accuracy when the measurements are taken with care.

Keywords: Mobile application; Participatory sensing; Secchi depth; Water quality

\section{Background}

Water is a necessity for life, and clean water is usually a sign of a healthy environment. Human activities can have adverse effects on water quality. For example, the use of fertilizers in agriculture can lead to increased nutrient levels in nearby waters and cause increased eutrophication. Thus, monitoring water quality is an essential part of taking care of the environment.

Water transparency is one commonly used indicator of water quality. It is mentioned as a supporting factor for the biological elements in the Water Framework Directive (WFD) (Solimini et al. 2006) and as one of the physicochemical quality elements for the ecological classification of coastal waters in the Marine Strategy Framework Directive (MSD) (Piha and Zampoukas 2011). High transparency values indicate clear water, whereas low transparency values indicate turbid or highly absorbing water, which usually means poor water quality. Water transparency defines how deep sun light will penetrate and hence the depth at which plants can grow.

\footnotetext{
* Correspondence: timo.toivanen@vtt.fi

${ }^{1}$ VIT Technical Research Centre of Finland, P.O. Box 1000, FI-02044 VIT Espoo, Finland

Full list of author information is available at the end of the article
}

A common measure of water transparency is Secchi depth. It is measured using a circular white (or black and white) plate, known as a Secchi disk, which is lowered into the water until it is no longer visible. Secchi disk measurements have previously been used in volunteer monitoring, e.g. in the Secchi Dip-In -program (http://www.secchidipin.org/), in which over 41,000 records of more than 7,000 separate water bodies have so far been uploaded into a data base through a web form.

The measurement of transparency with a Secchi disk has some limitations. First, the measurement is subjective and depends on the eyesight and ability of the person making the measurements. Second, it is affected by environmental factors such as waves and weather. Finally, it cannot be used in shallow water where the disk hits the bottom before disappearing. Transparency can also be measured horizontally with a Secchi disk or a black disk. With this method the measurement requires a periscope and is thus more complicated.

Other devices for the assessment of water transparency have also been developed. One example is the Transparency Tube. To take a measurement it is filled with water and slowly emptied until the markings at the 
bottom of the tube become visible when viewed from above. The measurement range of the tube is limited by the length of the tube (60 cm and $120 \mathrm{~cm}$ versions exist) and thus it is suitable only for low transparency waters such as rivers and streams.

Transparency can also be estimated by measuring turbidity. This requires a device that emits light and measures the amount of scattering from the sample. These turbidity meters are clearly more expensive than Secchi disks (in the range 800-1,000 USD).

The recent advances in mobile sensing and crowdsourcing provide potential means for collecting more data and observations from volunteers. Mobile phone sensing has become a hot topic in the areas of citizen science and crowdsourcing. Lane et al. (2010) presents four main reasons for the rapid increase in mobile sensing applications: 1) availability of cheap embedded sensors, 2) applications can be programmed to use these sensors, 3) app stores can be used to deliver new applications to large populations easily, and 4) back-end servers providing resources for computing.

Mobile sensing could extend the sensor networks of environmental institutes both spatially and temporally, and produce useful information for situation awareness, forecasting and scientific research. For example, D'Hondt et al. (2013) and Gallo and Waitt (2011) conclude that user-provided data can be used to gather useful datasets. Mobile sensing can be divided into two sensing paradigms (Lane et al. 2008). In opportunistic sensing the data collection is carried out automatically without actions by the users. Participatory sensing relies on the fact that users actively make and report their observations.

In this paper, we present a novel approach to water quality monitoring, called Secchi3000. This approach includes a participatory sensing application for mobile phones with which users take pictures of target areas inside a cheap and simple measurement device filled with water and send them with related metadata (e.g. location) to a central server. On the server, water turbidity and Secchi depth are automatically post-processed from the images using pattern recognition and computer vision approaches. The system was tested in the summer of 2012 and the results indicate that it provides a reliable method for water quality monitoring. Participatory water quality sensing can be a valuable tool either in providing measurement capabilities where none exist or in supplementing existing monitoring efforts with additional coverage. Also, Secchi3000 measurements can be used as an alert monitoring tool: if changes are detected in a water body, the authorities can send a professional water quality expert to assess the situation. In addition, the data collected using the system can be used e.g. in validation and completion of satellite image analysis.

\section{Methods}

\section{Overall system architecture}

The Secchi3000 water quality measurement system consists of the following parts: 1) mobile phone application for acquiring and sending observations, 2) Secchi3000 measurement device, 3) algorithms for analysing the water quality values from the images received, and 4) database, where all the data with analysed values are stored. The conceptual architecture of the Secchi3000 system is presented in Figure 1.

The mobile phone application the users can use to gather observations is called EnviObserver (Kotovirta et al. 2012). It is a tool for participatory sensing which utilizes people as sensors by enabling them to report environmental observations with a mobile phone. The current version of the Secchi3000 measurement device consists of a container and two measurement tags that are used in the analysis. The tags are located at different depths in order to derive water turbidity. After filling the container with water, the user takes a picture looking inside the container through a hole in the lid. GPS location and timestamp are automatically retrieved during the measurement. In addition to the picture, users can enter supplementary information, e.g. ID of the measurement site.

Finally, the user sends the data collected to a central server for automatic water quality analysis. The water quality analysis consists of two separate algorithms. After receiving a picture, the first algorithm automatically detects the locations of the tags in the picture and extracts pixel RGB values for the black, grey, and white areas of the two tags. The second algorithm carries out the actual water quality analysis based on the RGB values extracted by the tag recognition algorithm. After the automatic analysis, the result is sent back to the user and stored on a database.

\section{Data collection}

The system was tested in Finland during the summer of 2012. The test users were recruited by the Finnish Environment Institute and included people who collect water quality samples professionally, companies, water protection associations and private citizens. In the tests, 100 users sent 1,146 pictures for analysis. The samples were collected from lakes and coastal areas of Finland. The professional water quality experts took the Secchi3000 measurements during their routine measurement and sample collection trips. The other participants took measurements during their leisure activities mostly out of personal interest.

The results of the analysis are described in the next chapter. As the automatic analysis was not completely ready during the test period, the images received with related metadata were only stored in the database, and 

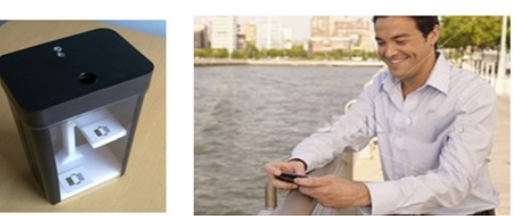

User with

Secchi3000

device

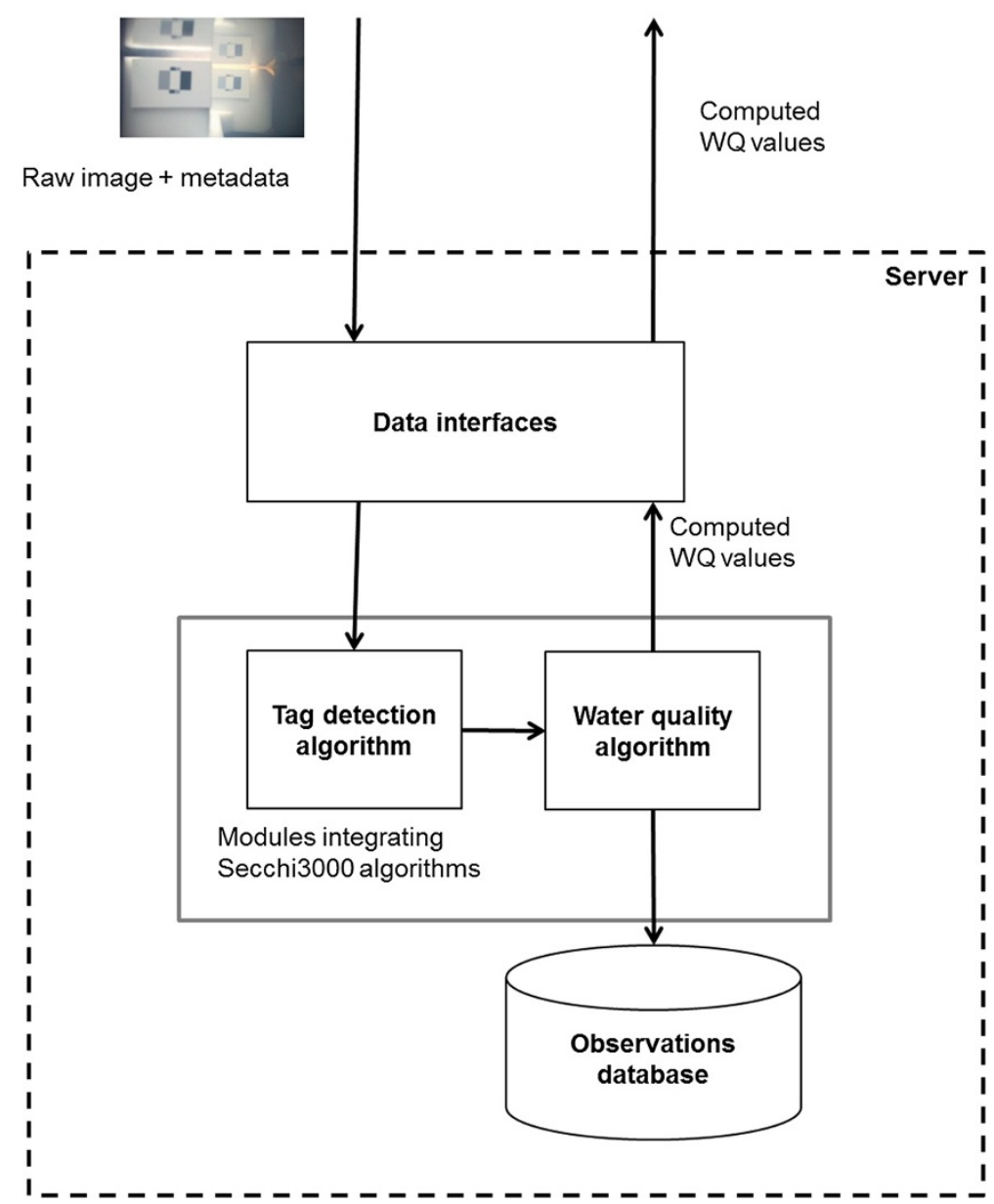

Figure 1 Conceptual architecture of the Secchi3000 system.

the analyses were carried out afterwards. The Secchi3000 devices were designed by the Finnish Environment Institute and VTT Technical Research Centre of Finland, and manufactured by a Finnish company. The system is currently being prepared for more large scale activities, including the commercial manufacture of the devices.

\section{Target detection}

Detecting accurate positions of white, grey and black targets in the images is challenging, because of many factors not related to the water quality:

- Varying illumination conditions create over-exposed areas, reflections and shadows in the inner structure of the measurement container. In the images, this translates into artefacts and undesired shapes that can occlude, extend or overlap with the target rectangles. A purely shape-based approach will not always be robust to this kind of artefacts.

- Standard cameras from average mobile phones deliver mid- to low-end image quality, with noise, blur, varying resolutions (often rather poor). This affects the sharpness of target rectangles in the image and therefore their detectability.

- Variability during acquisitions made by different human operators using various devices introduces undesired discrepancies; in particular, the acquisition angles of the camera with regards to the scene vary all the time. Because the acquisition system is used in-situ and not in a controlled environment, one cannot assume a priori either the position of targets in the image or their orientation. The target 
rectangles are not always oriented vertically or horizontally, they appear to be arbitrarily rotated in each image. Unlike in typical industrial computer vision applications, here the relative position and orientation of the camera and the scene cannot be known precisely.

On top of all these challenges, the lower the Secchi depth of the water is, the more difficult the target detection in the image. Because the water properties affect the reflectance and thus the pixel values, especially in cases of humic or very turbid waters, a target detection method based solely on colour or reflectance values in the image would most likely fail. Control points could be used to delineate the target areas; however, in turbid waters it is more reliable to detect larger homogeneous targets than control points.

For method development, a panel dataset of 20 images was manually chosen from a dataset of hundreds of water quality images acquired by users. The panel represents a wide variety of illumination conditions, water turbidity, and target detectability from the most favourable cases to the most difficult ones. Once fine-tuned on the panel, the method was applied to the whole image dataset for accuracy assessment.

The detection process was first to locate the surrounding areas of the lower and upper tags by the template matching method. The following processing is done in these two local tag areas (cf. Figure 2, the red rectangles are the areas detected using template matching).

The second step is to detect the white rectangle shape using a contour-based approach within the red rectangle area (the blue rectangle shape in Figure 2). The processing of the upper tag and lower tag is the same. The grey and black rectangle positions were obtained by similarity transformation. That is, by moving the rotated blue

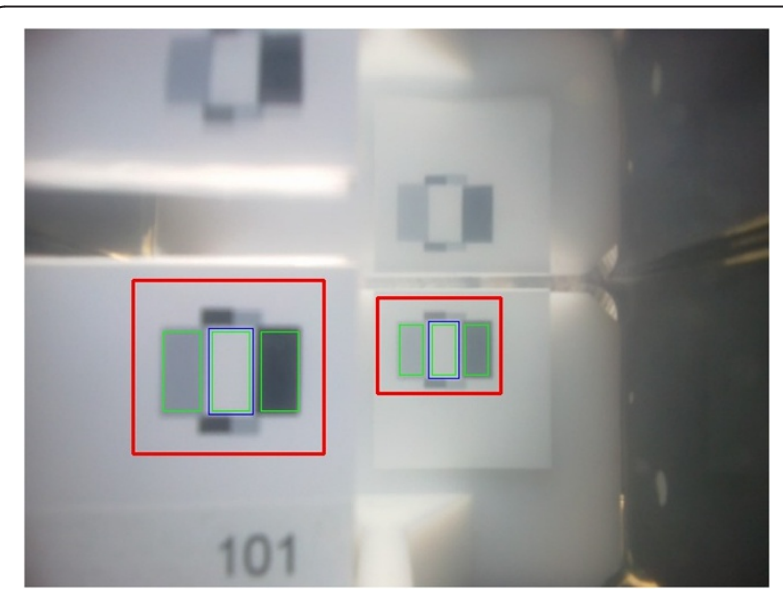

Figure $\mathbf{2}$ Locating the surrounding areas of lower and upper tags by the template matching method. rectangle leftward and rightward we get grey and black rectangles.

A mobile phone camera looking at the water tank through a hole approximates to a central projection in geometry computer vision. A central projection of a plane (tag) onto a parallel plane (image) is also called similarity transformation, which preserves parallelism, concurrence, ratio of division, etc. (Hartley and Zisserman 2004). That is angles between lines are not affected by rotation, translation or isotropic scaling. In particular parallel lines are mapped to parallel lines. The ratio of two lengths is an invariant. Similarly, a ratio of areas is an invariant.

When the image is extremely blurred and the camera is rotated, the white rectangle shape of the lower tag cannot be detected, but four corner image features can be extracted. Using the rectangle formed by these four corner feature points, the other two tags positions can be found, based on the similarity invariant.

\section{Results}

The results of the image analysis are shown in Figures 3 and 4. The Secchi3000 images were analysed both automatically and manually, and the results were compared against reference measurements. Our conclusions from the analysis are that the Secchi depth estimation works very well, while the turbidity estimates are a bit more scattered. Also, with larger turbidity values ( $>6 \mathrm{FNU}$ (Formazin Nephelometric Unit)) the difference from the reference value starts to increase (the order of magnitude is still correct).

The Secchi depth results shown in Figure 3 were derived from observations carefully made by one person using 3 mobile phones (Nokia E5, Nokia E7 and Nokia N500, depending on the location). The figure also shows that the difference between automatic and manual analysis is very small. Two to five observations were made with each phone from each location, and this explains the groups of data points visible in the image and gives an indication of the repeatability of the observations and differences between cameras. Overall, the variation of the measurements at each site was less than $10 \%$ of the reference value.

When the observations of the other test users are included in the results, there is significantly more scatter and the coefficient of determination is clearly lower. The likely explanations for this are: 1) the poor quality of the cameras in the mobile phones used by the test users (some were quite old models), 2) the deficient quality of the observations made by some of the users (the pictures sometimes included air bubbles, shadows and other error sources), 3) the difficulty of using the system in a rocking boat (as commented on by users), and 4) the lack of feedback from the system about the success of the measurement. The automated processing chain and the related feedback mechanism were implemented later based on 


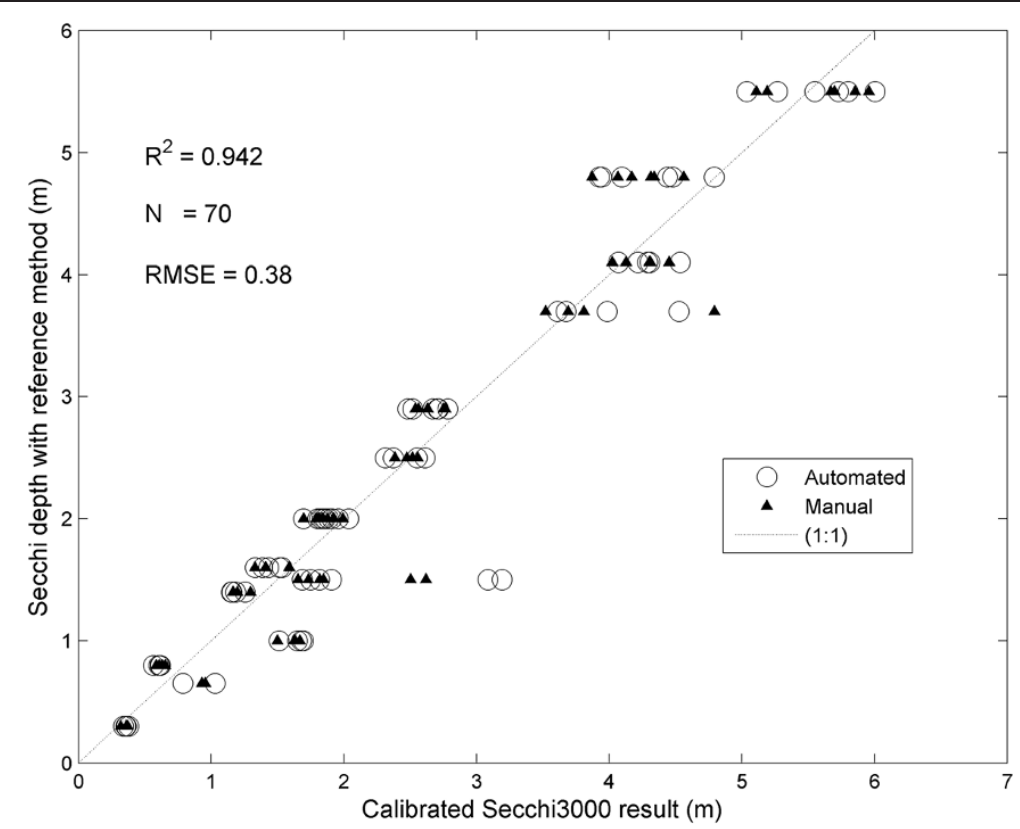

Figure 3 Secchi depth estimated using the Secchi3000 vs. reference method.

the user feedback. The average analysis time of the automatic processing is 2.09 seconds $(\mathrm{N}=1146)$ per image using a Pentium $43.2 \mathrm{GHz}$ processor with $4 \mathrm{~GB}$ memory.

\section{Discussion}

A measurement with the Secchi3000 has many advantages when compared to a measurement with a Secchi disk. For example, the measurement is not dependent on the eyesight of the user and the results are available quickly (with positioning) in a centralized system. Furthermore, the Secchi3000 measurement image can be re-analysed later if improved estimation algorithms become available. We are also planning to add more water quality parameters to be analysed automatically from the images such as total suspended matter (TSM) and absorption by humic substances (CDOM). This will give an indication of what

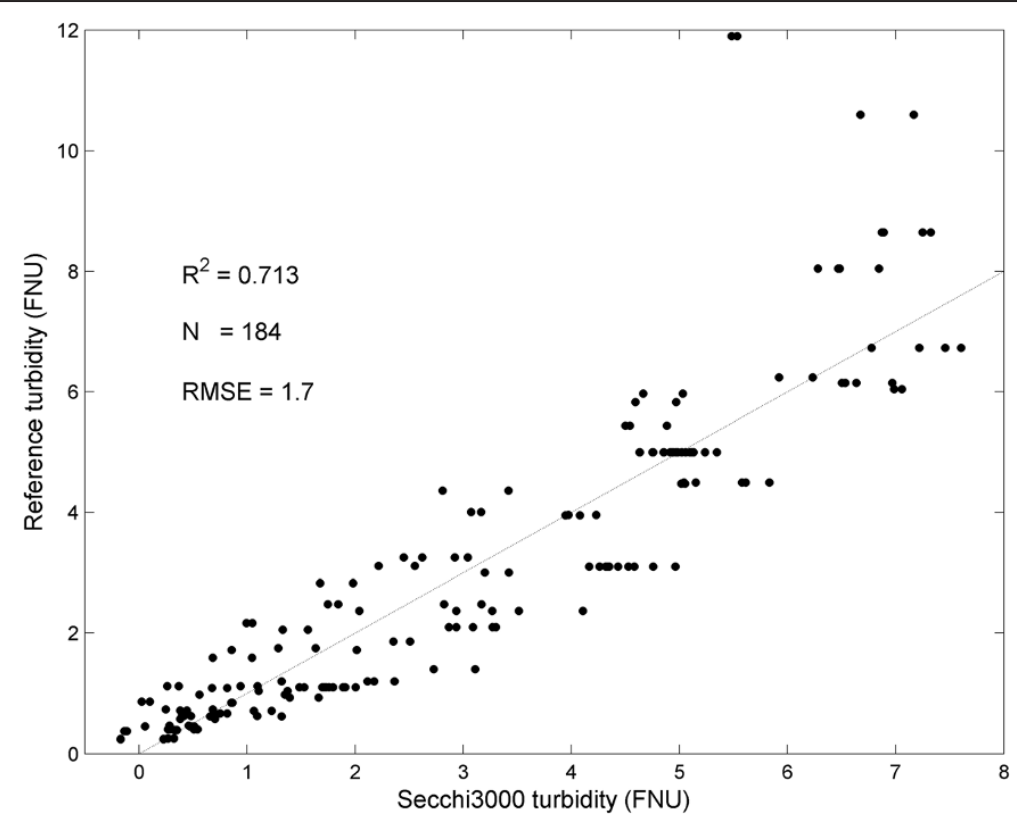

Figure 4 Turbidity estimated using the Secchi3000 vs. reference method. 
causes the reduced transparency instead of only giving an estimate of the value of the transparency.

When compared to Transparency Tube, the Secchi3000 has the advantage of a greater estimation range. The tubes are limited to a maximum transparency of $1.2 \mathrm{~m}$, while the Secchi3000 has successfully measured transparencies with a range from $0.3 \mathrm{~m}$ to over $5 \mathrm{~m}$.

The turbidity meters mentioned above are more accurate but also much more expensive and thus not suitable for participatory monitoring.

Although the methods presented in this paper work quite well, the real benefits for water quality management would come from a wide use of the system. Participatory sensing tools have proved to be technically feasible, but motivating potential observers to collect data is still an open issue which has been discussed by e.g. Reddy et al (2010) and Juong-Sik and Hoh (2010). The popularization of the Secchi3000 method would require a business model which enables the manufacture and distribution of the devices, improvements and maintenance of the mobile software for multiple mobile devices, and a way of motivating people to make regular observations in their neighbourhood.

It is still necessary to develop the system further in order to make the measurements easier and less prone to errors. For example, the immediate delivery of the result of the measurement to the user, which has now been implemented, will help in reducing the number of poor quality observations. Also, the user instructions need to be improved and possibly moved into the app (the app should guide the user in making the measurement). In addition to the Secchi3000 Java ME mobile application that was used in data gathering during the summer of 2012, versions of the mobile phone application are now available for iOS, Android and Windows platforms. This greatly increases the potential user base of the system, which is essential for popularizing the method. The current price of a Secchi3000 measurement device is slightly higher than the cost of a Secchi disk. Presumably, this will change with larger scale production of the devices.

\section{Conclusions}

In this paper, we have presented a novel approach to automatic water quality analysis based on images from the Secchi3000 measurement device filled with water. The EnviObserver participatory sensing tool was used for collecting the data from users and for analysing the data received.

For tag detection, template matching can accurately locate the tags in an image with different size and orientation and separate them from clutter (such as tag reflection, text and the tag platform borders). Using image feature- and shape-based recognition increased the accuracy and robustness of the tag positions.
Results show that, overall, the system performs well. Both Secchi depth and turbidity are estimated with excellent or good accuracy when the measurements are taken with care. However, further development of the system is needed to ease the making of observations, and user instructions need to be improved so that users are able to provide better quality images for analysis.

\section{Competing interests}

The authors declare that they have no competing interests.

\section{Authors' contributions}

TT implemented the system architecture and drafted the manuscript, which all the authors helped to edit and polish. SK developed the algorithm for water quality estimation using target RGB-values extracted from mobile phone images and compared the measurements against reference measurements. VK provided domain-related guidance and helped design the system. MM and PC implemented the target detection algorithm. All the authors read and approved the final manuscript.

\section{Acknowledgements}

The work was supported by the Measurement, Monitoring and Environmental Assessment research programme funded by Tekes, the Finnish Funding Agency for Technology and Innovation. We are grateful to the Maa- ja vesitekniikan tuki ry for financial support.

\section{Author details}

${ }^{1}$ VTT Technical Research Centre of Finland, P.O. Box 1000, FI-02044 VTT Espoo, Finland. ${ }^{2}$ Finnish Environment Institute, P.O. Box 140, Helsinki, Finland.

Received: 27 August 2013 Accepted: 17 October 2013

Published: 18 October 2013

\section{References}

D'Hondt E, Stevens M, Jacobs A (2013) Participatory noise mapping works! An evaluation of participatory sensing as an alternative to standard techniques for environmental monitoring. Perv Mobile Comp 9(5):68-694. ISSN 1574-1192, doi:10.1016/j.pmcj.2012.09.002

Gallo T, Waitt D (2011) Creating a successful citizen science model to detect and report invasive species. Bio Sci 61(6):459-465. 10.1525/bio.2011.61.6.8

Hartley R, Zisserman A (2004) Multiple View Geometry in Computer Vision, 2nd edition. Cambridge University Press, Cambridge. ISBN 0521540518

Juong-Sik L, Hoh B (2010) Dynamic pricing incentive for participatory sensing. Perv Mobile Comp 6(6):693-708

Kotovirta V, Toivanen T, Tergujeff R, Huttunen M (2012) Participatory sensing in environmental monitoring - experiences. Proc Sixth Int Conf Innov Mobile Int Serv Ubiquit Comp (IMIS '12). doi:10.1109/IMIS.2012.70

Lane ND, Eisenman SB, Musolesi M, Miluzzo E, Campbell AT (2008) Urban sensing systems: opportunistic or participatory? In: Proceedings of the 9th workshop on Mobile computing systems and applications (HotMobile '08). ACM, New York, NY, USA. 10.1145/1411759.1411763, doi: 10.1145/1411759.1411763

Lane ND, Miluzzo E, Hong L, Peebles D, Choudhury T, Campbell AT (2010) A survey of mobile phone sensing. Comm Mag 48(9):140-150. doi:10.1109/ MCOM.2010.5560598

Piha H, Zampoukas N (2011) Review of Methodological Standards Related to the Marine Strategy Framework Directive Criteria on Good Environmental Status. Publications Office of the European Union, Luxembourg. doi:10.2788/60512

Reddy S, Estrin D, Srivastava M (2010) Recruitment framework for participatory sensing data collections. Proceedings of the 8th International Conference on Pervasive Computing, Helsinki, Finland

Solimini A, Cadroso A, Heiskanen A (ed) (2006) Indicators and methods for the ecological status assessment under the Water Framework Directive. Office for Official Publications of the European Communities, Luxembourg. ISBN 92-79-02646-1

doi:10.1186/2193-2697-2-9

Cite this article as: Toivanen et al:: Water quality analysis using an inexpensive device and a mobile phone. Environmental Systems Research 2013 2:9. 